\title{
RESEARCH HIGHLIGHT Immunological memory: ILC1s come into view
}

\author{
Yawen Chen ${ }^{1,2}$, Zhigang $\operatorname{Tian}^{1,2}$ and Hui Peng ${ }^{1,2}$ \\ Cellular \& Molecular Immunology (2019) 16:895-896; https://doi.org/10.1038/s41423-019-0311-4
}

Immunological memory is traditionally thought to be an important property that distinguishes adaptive immunity from innate immunity. Unlike $T$ and $B$ lymphocytes, innate lymphoid cells (ILCs) lack rearranged antigen receptors. However, increasing evidence implies that ILCs are capable of conferring antigenspecific memory responses in different experimental models. Among these models, natural killer (NK) cell-mediated memory responses during mouse cytomegalovirus (MCMV) infection have been extensively studied. ${ }^{1}$ The generation of MCMV-specific memory NK cells is dependent on the recognition of MCMVencoded glycoprotein m157 by the activating receptor $\mathrm{Ly}_{49 \mathrm{H}}{ }^{2}$ MCMV-induced memory NK cells circulate in the blood. In contrast to circulating NK cells, other subsets in the ILC family are mostly tissue-resident cells. ${ }^{3}$ Whether tissue-resident ILCs mediate MCMVspecific memory responses is unclear. Recently, Weizman et al. ${ }^{4}$ reported that ILC1s can confer antigen-specific memory responses in the liver during MCMV infection (Fig. 1).

As group $1 \mathrm{ILCs}$, both circulating NK cells and tissue-resident ILC1s express the pan-NK cell markers NK1.1 and NKp46 and have the ability to produce interferon gamma (IFN- $\gamma){ }^{5}$ Approximately $50 \%$ of circulating NK cells express $\mathrm{Ly} 49 \mathrm{H}$, which plays an important role in host defense against MCMV infection. Despite lacking Ly49H expression, ILC1s respond rapidly to IL-12 secreted by local dendritic cells during MCMV infection. ${ }^{6}$ In addition, they are the major producers of IFN- $\gamma$ at early time points prior to circulating NK cell responses ${ }^{6}$ and thereby contribute to early host protection. Moreover, in contrast to widely distributed circulating NK cells, ILC1s are preferentially distributed in nonlymphoid tissues. ${ }^{7}$ In particular, liver ILC1s (also termed liver-resident NK cells) account for a substantial fraction of liver group 1 ILCs. These cells can recall different haptens in contact hypersensitivity, ${ }^{8}$ in which memory ILC1s may dynamically migrate, as the sensitization and challenge processes occur at different sites. ${ }^{9}$ During pathogen infection, tissue-resident memory $T$ cells that are generated and persist at a regional site of infection combine with circulating memory $\mathrm{T}$ cells to provide important protective immunity. ${ }^{10}$ Therefore, it is important to investigate whether tissue-resident memory ILCs co-exist with circulating memory NK cells. Such investigations will lead to a morecomprehensive understanding of the coordination between local and systemic immunity.

The memory formation process of adaptive immune cells and circulating NK cells can be divided into three phases: robust expansion, contraction and persistent memory. ${ }^{11}$ Weizman et al. ${ }^{4}$ found that the number of liver ILC1s initially decreased during the first 2 days after MCMV infection. Subsequently, expansion was observed between days 2 and 7, and this expansion was followed by the contraction phase. After the resolution of MCMV infection, liver ILC1s exhibited long-term persistence.

Molecular mechanisms responsible for the expansion of circulating NK cells in response to MCMV infection provide clues for the further study of ILC1 responses. Experiments with mixed bone marrow chimeric mice showed that, similar to circulating NK cells, the accumulation of liver ILC1s at day 7 post MCMV infection also required the proinflammatory cytokines interleukin-12 (IL-12) and IL-18, as well as the transcription factor Zbtb32. However, the Ly49H-m157 interaction is critical for MCMV-induced proliferation of circulating NK cells but not for that of ILC1s, consistent with the lack of Ly49H expression on ILC1s. During MCMV infection, liver ILC1s maintained their lineage stability and tissue-resident properties, suggesting the absence of interconversion between ILC1s and circulating NK cells.

Similar to memory T lymphocytes, MCMV-experienced liver ILC1s exhibited upregulated expression of cytokine receptors, including IL-7Ra, IL-12Ra and IL-18R. Weizman et al. further demonstrated that a portion of naive $\mathrm{IL}_{-} 18 \mathrm{R}^{-} \mathrm{ILC} 1 \mathrm{~s}$ acquired stable expression of IL-18R after infection. These findings raised the possibility that IL-18 expression might mark an ILC1 population with memory-like properties. Indeed, transcriptional and epigenetic profiling revealed that IL- $18 \mathrm{R}^{+} \mathrm{ILC} 1 \mathrm{~s}$ showed a bias toward the expression of memory signature genes after infection. More importantly, compared with IL-18R $\mathrm{R}^{-} \mathrm{ILC} 1 \mathrm{~s}$ from naive or MCMV-infected mice, MCMV-induced IL-18R ${ }^{+} \mathrm{ILC} 1 \mathrm{~s}$ had an enhanced ability to produce IFN- $\gamma$ in response to stimulation via the activating receptor NK1.1 (Nkrp1c) or secondary infection. This finding implied the existence of memory-like ILC1s induced by MCMV infection.

The immunological memory of adaptive lymphocytes depends upon a substantially diverse repertoire of T- or B-cell receptormediated specific recognition of antigens. Previous studies have demonstrated that prior stimulation with the cytokines IL-12, IL-15, and IL-18 can enhance the effector function of NK cells after secondary exposure. ${ }^{12}$ Moreover, IL-33 can induce the generation of memory-like ILC2s $\mathrm{s}^{13}$ in an antigen-independent manner. Weizman et al. initially established heterologous infection models by infecting MCMV-primed mice with influenza to assess whether IL-18R ${ }^{+}$ILC1-mediated recall responses to MCMV were antigendependent. They found that enhanced production of IFN- $\gamma$ by lung ILC1s did not occur in this model. Furthermore, upon ex vivo stimulation with IL-12 and IL-18, MCMV-experienced liver IL-18R ${ }^{+}$ ILC1s produced IFN- $\gamma$ at a level comparable to that observed in uninfected mice. These findings suggest that the enhanced effector function of IL-18R ${ }^{+}$ILC1s during MCMV reinfection is driven by MCMV-specific signals.

\footnotetext{
'Division of Molecular Medicine, Hefei National Laboratory for Physical Sciences at the Microscale, the CAS Key Laboratory of Innate Immunity and Chronic Disease, School of Life

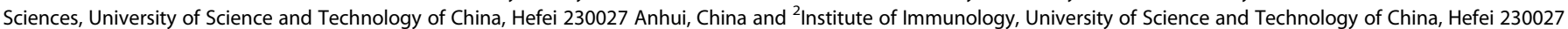
Anhui, China
}

Correspondence: Hui Peng (huipeng@mail.ustc.edu.cn)

Received: 22 September 2019 Accepted: 25 September 2019

Published online: 28 October 2019 


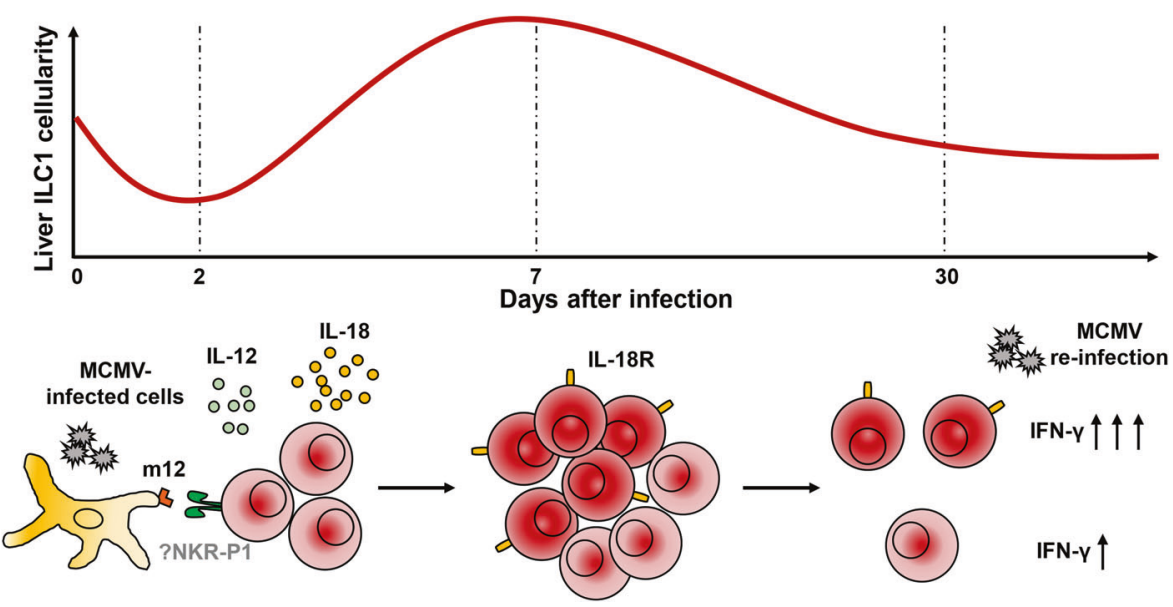

Fig. 1 Liver IL-18R $\mathrm{R}^{+}$ILC1s mediate the recall response to MCMV infection in a MCMV-encoded m12-dependent manner. Liver ILC1s proliferate and increase IL-18R expression after MCMV-m12 infection. The inflammatory cytokines IL-12 and IL-18 play an important role in this process. After going through the contraction phase, IL-18R $\mathrm{R}^{+} \mathrm{ILC} 1 \mathrm{~s}$ exhibit enhanced IFN $\gamma$ production upon secondary exposure to MCMV-m12

The production of IFN- $\gamma$ is increased by MCMV-induced IL- $18 \mathrm{R}^{+}$ ILC1s upon cross-linking of NK1.1. In addition, MCMV-encoded glycoprotein $\mathrm{m} 12$ can interact with NK1.1 as well as with two other NKR-P1 receptors (i.e., Nkrp1a and Nkrp1b). ${ }^{14}$ Considering these observations, Weizman et al. investigated whether the recall of MCMV by ILC1s is mediated by the recognition of $\mathrm{m} 12$. They infected mice with three MCMV strains, including the MCMV-Smith strain as the control, an MW97 mutant strain deficient in $\mathrm{m} 12$ $(\Delta \mathrm{m} 12)$ and an $\mathrm{m}_{12} 2^{\text {smith }}$ strain that expressed an $\mathrm{m} 12$ ortholog in the MW97 mutant strain. Only infection with $\Delta \mathrm{m} 12$ failed to induce the expansion of $\mathrm{IL}-18 \mathrm{R}^{+} \mathrm{ILC} 1 \mathrm{~s}$ in the liver. Unlike IL-18R $\mathrm{R}^{+}$ ILC1s from $\Delta \mathrm{m} 12$-infected mice, IL-18R ${ }^{+}$ILC1s from MCMV-Smithor $\mathrm{m} 12^{\text {smith }}$-infected mice displayed increased production of IFN- $\gamma$ upon cross-linking of NK1.1. Consistent with these results, $\Delta \mathrm{m} 12$ primed $R a g 2^{-1-}$ mice exhibited increased viral titers following MCMV-Smith challenge compared with those in the groups primed with the m12-expressing strains. These results suggest that $\Delta \mathrm{m} 12$-primed $\mathrm{IL}-18 \mathrm{R}^{+} \mathrm{ILC} 1 \mathrm{~s}$ do not mount effective recall responses sufficient to confer host protection.

In summary, the study conducted by Weizman et al. highlights the generation of liver IL-18R ${ }^{+} \mathrm{ILC} 1 \mathrm{~s}$ with memory properties during MCMV infection in a viral antigen-dependent manner. These findings provide new evidence regarding ILC1 memory and further advance the understanding of innate immune memory. However, further investigations are warranted. It is important to identify the receptor that engages with the $\mathrm{m} 12$ glycoprotein, which is indispensable for the IL- $18 \mathrm{R}^{+}$ILC1 memory response. Both NK1.1 and Nkrp1a are established activating receptors that could interact with $\mathrm{m} 12$; however, their importance in this process is not clear. Given that these receptors are widely expressed in circulating NK cells and ILC1s, whether $\mathrm{m} 12$ is involved in circulating NK cell memory remains to be investigated. Studies have demonstrated that memory ILC1s that recall haptens and certain viruses preferentially reside in the liver. ${ }^{8,9,15,16}$ Whether the enhanced secondary effector functions of ILC1s in response to MCMV infection are restricted to the liver or also occur in other tissues remains unclear. Moreover, it is necessary to evaluate the importance of the ILC1-mediated recall response to viral infections in immunocompetent hosts. Overall, despite the unanswered questions, investigation of the memory functions of ILC1s increases our understanding of basic ILC1 biology and may provide additional insights into vaccine development strategies.

\section{ADDITIONAL INFORMATION}

Competing interests: The authors declare no competing interests.

\section{REFERENCES}

1. Cerwenka, A. \& Lanier, L. L. Natural killer cell memory in infection, inflammation and cancer. Nat. Rev. Immunol. 16, 112-123 (2016).

2. Sun, J. C., Beilke, J. N. \& Lanier, L. L. Adaptive immune features of natural killer cells. Nature 457, 557-561 (2016).

3. Gasteiger, G., Fan, X., Dikiy, S., Lee, S. Y. \& Rudensky, A. Y. Tissue residency of innate lymphoid cells in lymphoid and nonlymphoid organs SD. Science 350, 981-985 (2015).

4. Weizman, O.-E., Song, E., Adams, N. M., Hildreth, A. D., Riggan, L. \& Krishna, C. et al. Mouse cytomegalovirus-experienced ILC1s acquire a memory response dependent on the viral glycoprotein m12. Nat. Immunol. 20, 1004-1011 (2019).

5. Peng, H. \& Sun, R. Liver-resident NK cells and their potential functions. Cell Mol. Immunol. 14, 890-894 (2017).

6. Weizman, O.-E., Adams, N. M., Schuster, I., Krishna, C., Pritykin, Y. \& Lau, C. et al. ILC1 confer early host protection at initial sites of viral infection. Cell 171, 795-801 (2017).

7. Fan, X. \& Rudensky, A. Y. Hallmarks of tissue-resident lymphocytes. Cell 164, 1198-1211 (2016).

8. Peng, H., Jiang, X., Chen, Y., Sojka, D. K., Wei, H. \& Gao, X. et al. Liver-resident NK cells confer adaptive immunity in skin-contact inflammation. J. Clin. Invest 123, 1444-1456 (2013).

9. Wang, X., Peng, H., Cong, J., Wang, X., Lian, Z. \& Wei, H. et al. Memory formation and long-term maintenance of IL-7Ra ${ }^{+}$ILC1s via a lymph node-liver axis. Nat. Commun. 9, 1-12 (2018).

10. Schenkel, J. M. \& Masopust, D. Tissue-resident memory T cells. Immunity 41, 886-897 (2014).

11. O'Sullivan, T. E., Sun, J. C. \& Lanier, L. L. Natural killer cell memory. Immunity $\mathbf{4 3 ,}$ 634-645 (2015).

12. Cooper, M. A., Elliott, J. M., Keyel, P. A., Yang, L., Carrero, J. A. \& Yokoyama, W. M. Cytokine-induced memory-like natural killer cells. Proc. Natl. Acad. Sci. USA 106, 1915-1919 (2009)

13. Martinez-Gonzalez, I., Mathä, L., Steer, C. A., Ghaedi, M., Poon, G. F. T. \& Takei, F. Allergen-experienced group 2 innate lymphoid cells acquire memory-like properties and enhance allergic lung inflammation. Immunity 45, 198-208 (2016).

14. Aguilar, O. A., Berry, R., Rahim, M. M. A., Reichel, J. J., Popović, B. \& Tanaka, M. et al. A viral immunoevasin controls innate immunity by targeting the prototypical natural killer cell receptor family. Cell 169, 58-71 (2017).

15. Paust, S., Gill, H. S., Wang, B. Z., Flynn, M. P., Moseman, E. A. \& Senman, B. et al. Critical role for the chemokine receptor CXCR6 in NK cell-mediated antigenspecific memory of haptens and viruses. Nat. Immunol. 11, 1127-1135 (2010).

16. Li, T., Wang, J., Wang, Y., Chen, Y., Wei, H. \& Sun, R. et al. Respiratory influenza virus infection induces memory-like liver NK cells in mice. J. Immunol. 198, 1242-1252 (2017). 